\title{
Two and Half Years with Dabigatran Etexilate in Neurological Units
}

\author{
Jerzy Krupinski ${ }^{1,2 *}$, Sonia Huertas ${ }^{1,2}$ and M Angels Font ${ }^{2,3}$ \\ ${ }^{1}$ Department of Neurology, Hospital Universitari Mútua Terrassa, Terrassa, Spain \\ ${ }^{2}$ Fundació per a Recerca i Docencia Mútua Terrassa, Terrasa, Spain \\ ${ }^{3}$ Department of Neurology, Consorci Sanitari de Terrassa, Terrassa, Spain
}

Atrial fibrillation (AF) is the most frequently clinically significant cardiac arrhythmia, and is an important independent risk factor for thrombo embolic events. The prevalence of AF increases with age, with less than $1 \%$ among people under 60 years, $5 \%$ at age 65 years, and more than $9 \%$ estimated in people over 80 years. AF is estimated to affect more than 6 million patients in Europe and approximately 2.3 million patients in the U.S., and with the aging population the number of patients with $\mathrm{AF}$ is increasing. $\mathrm{AF}$ is associated with a 4-5 fold risk of ischemic stroke and is up to $16 \%$ of all strokes and $30 \%$ of strokes in patients over 80 years of age. Furthermore, there is evidence that strokes associated with $\mathrm{AF}$ are more disabling than those not associated with FA [1-8]. Treatment with oral anticoagulation (ACO) is very effective in preventing stroke in patients with $\mathrm{AF}$ [9]. The vitamin $\mathrm{K}$ antagonists (VKA) have been the standard of care in recent decades. The new ACOs (NACOs) by their efficacy / safety profile and no-need of routine monitoring will replace in the near future warfarin / acenocumarol [10]. Meanwhile, dabigatran is a direct and selective potent inhibitor of thrombin, and unlike the AVK, can be administered without the need for routine monitoring of anticoagulation. The RE-LY trial demonstrated conclusively and categorically, that the dose of $150 \mathrm{mg}$ twice daily of dabigatran was superior to warfarin in reducing stroke and systemic embolism, providing a risk reduction of 35\% (RR 0.65 , $95 \% \mathrm{CI}, 0.52$ to $0.81, \mathrm{P}<0.001)$ further to the reduction obtained with warfarin $[11,12]$. One of the most interesting findings from the RE-LY was documenting a significant RRR in the rate of cerebral bleeding with both doses of dabigatran compared with warfarin (RRR 74\%, p <0.001 and RRR 69\%, p <0.001, dose $110 \mathrm{mg}$ BID and $150 \mathrm{mg}$ respectively). In the three major clinical trials of new anticoagulants patients were introduced between 7-14 days post stroke. The waiting was explained to avoid earlier bleeding complications. In the case of dabigatran, the inclusion has been 14 days post stroke. Several clinical factors (other antithrombotic treatments) are associated with an increased risk of hemorrhagic transformation after ischemic stroke: stroke size, patient age, other concomitant medication (antihypertensive drugs, ant diabetic), previous stroke, small petechiae in the area appearing early post ischemic stroke and cardio embolic stroke. In most cases, symptomatic intracranial hemorrhage $(\mathrm{SICH})$ appears between 7 and 10 days post stroke. However, in clinical practice, many patients initiate anticoagulant treatment between 4-7 days post stroke. In some centres, treatment is started with bridging both low weight heparins or heparin sodium and AVKs in the first 24-48 hours post stroke. It seems logical that patients with TIA or minor stroke might benefit from early treatment with new anticoagulants without increasing bleeding risk. New scales as $\mathrm{CHADS}_{2}, \mathrm{CHA}_{2} \mathrm{DS}_{2}$-VASc, HAS-BLED can help stratify risk of cerebral or systemic hemorrhage. Furthermore, neuroimaging study with gradient echo MRI can detect presence of petechiae zones associated with ischemia. It is likely that dabigatran may be justified to use a few days to two weeks post stroke based on risk of recurrent stroke and low risk of bleeding. However, clinical and biochemical data is missing in this population and would be of great interest and study. Generally, older patients have a higher risk of embolism by AF and most important independent predictor for having a stroke is to have had a previous stroke or TIA. In the RE-LY study only $19.9 \%$ of patients had a previous stroke and $\mathrm{CHADS}_{2}$ through the study was 2.1. The efficacy / safety did not change in patients with previous stroke or not. Typical neurological patient is a patient with a high $\mathrm{CHADS}_{2}$ and previous stroke. It would be of interest to confirm in this group of patients the benefit of early treatment with dabigatran.

Currently, dabigatran etexilate is approved for the prevention of stroke or systemic embolism in patients with nonvalvular AF. Dabigatran is approved in more than 80 countries with clinical experience in over $1,000,000$ patients worldwide. Dabigatran has the profile of an ideal anticoagulant for a clinical neurologist: efficacy at least equal to that of warfarin, higher if $150 \mathrm{mg}$ bid, predictable response, wide therapeutic window, low incidence and severity of adverse effects, oral fixed dose without the need routine control of anticoagulation, low potential for food or drug interactions, start and end fast action [13].

Importantly, the NACOs reduce mortality compared to warfarin. In the 3 studies combined 50,578 patients, 28,342 patients were randomized to NACOs and 22,236 to warfarin. The first combined analysis including the RE-LY study arm with $110 \mathrm{mg}$ of dabigatran showed NACOs significantly reduced all-cause mortality by $10 \%$ compared with dose-adjusted warfarin $(\mathrm{HR}=0.90,95 \% \mathrm{CI}: 0.84-0.96$, $\mathrm{P}=0.002)$, with no significant heterogeneity $(\mathrm{P}=.86)$. In the same vein, the second combined analysis, including the RE-LY study arm with $150 \mathrm{mg}$ of dabigatran showed NACOs significantly reduced allcause mortality by $9 \%$ compared with dose-adjusted warfarin (HR = $0.91,95 \% \mathrm{CI}: 0.85-0.97, \mathrm{P}=0.004)$, with no significant heterogeneity $(\mathrm{P}=0.91)[14]$.

We present the analysis of our experience in the use of dabigatran etexilate in clinical practice in our center. We discuss the safety and efficacy after 2.5 years in 172 patients followed for at least 1 year. In addition, we highlight some of practical that aspects can still be open to discussion in our patients with stroke.

Since the approval of dabigatran use by the FDA, we included consecutively patients in our hospital with non-valvular AF and ischemic stroke without significant renal impairment (creatinine clearance $>30$ $\mathrm{ml} / \mathrm{min} / 1,73 \mathrm{~m}^{2}$ ), and with an indication for anticoagulation. Before European approval, all patients signed informed consent. Before starting the treatment was explained to the patient and family, gave the patient card and performed outpatient report recommending perform

*Corresponding author: Jerzy Krupinski, Department of Neurology, Hospital Universitari Mútua Terrassa (Barcelona) PI, Dr. Robert, 5; 08221 Terrassa (Barcelona), Spain, E-mail: jkrupinski@mutuaterrassa.es

Received August 07, 2013; Accepted August 14, 2013; Published August 16 , 2013

Citation: Krupinski J, Huertas S, Angels Font M (2013) Two and Half Years with Dabigatran Etexilate in Neurological Units. J Vasc Med Surg 1: e112. doi: 10.4172/2329-6925.1000e112

Copyright: @ 2013 Krupinski J, et al.. This is an open-access article distributed under the terms of the Creative Commons Attribution License, which permits unrestricted use, distribution, and reproduction in any medium, provided the original author and source are credited. 
renal function control on 6-12 months follow-up. In the follow-up assessment between 6 and 12 months after initiation of treatment with dabigatran, blood tests were taken and patients were asked for potential adverse drug effects. We reviewed the medical records for potential primary effects not reported and consulted the database of hospital pharmacy. In total, 172 patients were evaluated, aged between 36 and 94 years (74 males and 98 females). 59\% were older than 80 years with an average CHAD2DS2-VASc -5.1. Sixty-five patients underwent a change of Sintrom to Pradaxa and 117 were de novo patients. In 55 patients, FA was diagnosed de novo. The ischemic risk assessed CH2DS2-VASc scale was 5.1 on average in our population. The estimated bleeding risk HAS-BLED scale was 3.9. In 26 patients the creatinine clearance prior to initiation of treatment was $30-60 \mathrm{ml} / \mathrm{min} / 1,73 \mathrm{~m}^{2}$.

Different types of adverse events $(n=60)$ were produced in a total of 22 patients $(12.8 \%)$. Blood tests in monitoring showed no deterioration of renal function, except in two patients. One was diagnosed underlying systemic disease and subsequent cerebral stroke and the other was a patient of advanced age and multiple comorbidities. In the two patients was decided to withdraw the drug. The mean coagulation parameters were as follows: Prothrombin time (PT) 68\%, TP (ratio) 1.4, INR 1.6. Three patients had recurrence of ischemic stroke. Two of them in context of treatment failure and one patient was diagnosed with systemic disease, lupus. One patient experienced intracranial hemorrhage: subdural hematoma as in context of accident. In this patient, NACOs was stopped and patient had good recovery. Another patient had a spontaneous intracerebral hematoma. The patient was underweight, $<50 \mathrm{~kg}$, multiple comorbidities and concomitant treatments and outcome was fatal. Other bleedings appeared in 14 subjects, including 7 rectal, 3 hematuria, 2 hempoptisis, and 2 epistaxis. Dyspepsia was reported in 3 patients, and two had to withdraw the drug. In 3 patients reported anemia. Other adverse events reported also in the RE-LY study were generally lower than those reported in the clinical trial. Over 1 year drug was withdrawn in 33 patients. This number is higher than that reported in the RE-LY trial. In most of these patients withdrawal was not motivated by adverse event, but medical decision making follow-up (GP, hematologist) and decided to change to sintrom treatment. There were no serious or fatal bleeding except in one patient described above, who suffered from brain hemorrhage. In our group, prior to initiating treatment with dabigatran 36 patients received iv rt-PA and / or mechanical thrombectomy and there were no further complications.

It is clear that treatment with dabigatran and generally NACOs raise new possibilities for vascular neurology.

One of the questions that are still open is when to start anticoagulation after stroke. The Cochrane database contains 24 studies $(\mathrm{n}=23,748$ patients). It analyses the use of various anticoagulants in the first 48 hours after stroke: unfractionated heparin (89\%), low molecular weight heparin, heparinoids, ACO, and thrombin inhibitors. In 11 studies $(n=22,776)$, there is no reduction in the likelihood of death or be independent, whatever the cause, by the fact anticoagulation started early. There was little stroke recurrence, but increased intracranial hemorrhage ( $\mathrm{ICH})$ symptomatic, although reducing the frequency of pulmonary embolisms, but benefit was nullified by an increase in extracranial bleeding [15].

There are also several studies examining the use of anticoagulation in the acute phase of stroke. In the double-blind study HAEST (Heparin in Acute Embolic Stroke Trial), there was a reduction in early recurrence of ischemic stroke in $\mathrm{AF}$ patients randomized to receive dalteparin vs aspirin. Instead, the study IST (International Stroke Trial) showed a clear reduction in dose-dependent and recurrent ischemic stroke in patients receiving subcutaneous heparin. However, the reduction of early recurrence of ischemic stroke with heparin in the IST study was virtually nullified by increased symptomatic intracerebral hemorrhage. In the RAPID study (Rapid Anticoagulation Prevents Ischemic Damage) patients receiving unfractionated heparin suffered a minor stroke recurrence and a similar incidence of serious bleeding events than patients who received aspirin. The secondary hemorrhagic transformation usually occured between 12 hours and 4 days after the onset of stroke. Authors concluded that it seems reasonable starting warfarin therapy as soon as the patient is medically and neurologically stable, often 2 or 3 days after the onset of stroke to achieve a therapeutic anticoagulation between 7 and 10 days after the onset of stroke [16].

The next question is when to launch dabigatran after ischemic stroke. Three large clinical trials of NACOs included patients between 7 and 14 days post stroke. The wait was justified to prevent bleeding complications early. In the case of dabigatran inclusion was 14 days post stroke. Several clinical factors (other antithrombotic treatments) are associated with an increased risk of hemorrhagic transformation after ischemic stroke: stroke size, patient age, other concomitant medication (antihypertensive, antidiabetic), prior history of stroke, the presence of small petechiae in the early post ischemic stroke, or cardioembolic stroke. Anyway, in clinical practice, in many patients with stroke anticoagulant treatment is started between 4 and 7 days post stroke. In some centers, treatment with both low weight heparins and heparin sodium in the first 24-48 hours post stroke is used as bridge therapy. It seems logical that patients with TIA or minor stroke might benefit from early treatment with new anticoagulants without increasing the risk of bleeding. New scales as CHADS2, CHA2DS2-Vasc, HAS-BLED can help stratify the risk of stroke or systemic bleeding. Furthermore, the neuroimaging study with gradient echo MRI can detect the presence of small bleeding associated with areas of ischemia or other old microbleeds. Currently, it is recommended that dabigatran may be justified to use a few days to two weeks post stroke based on the high risk of recurrent stroke and low risk of bleeding. Clinical and biochemical data is missing in this population would be of great interest and study. Generally, older patients are at increased embolic risk for AF and most important independent predictive factor for having a stroke is to have had a previous stroke or TIA. In the RE-LY study only $19.9 \%$ of patients had a previous stroke and through study CHADS2 was 2.1. The efficacy / safety did not differ in patients with previous stroke or not. Given that the typical neurological patient is a patient with a high CHADS2 and previous stroke, it is welcome to confirm the benefit of early treatment with dabigatran [17-20].

Another important aspect to consider is the secondary prevention of ischemic stroke after intracerebral hemorrhage related to ACOs. It is imperative to assess very carefully the risk / benefit ratio. Regarding hypertensive vasculopathy, the recurrence risk of hemorrhagic stroke is $2.1 \%$ at 2 years. However, with regard to amyloid angiopathy, the risk of hemorrhagic stroke recurrence is $22 \%$ at 2 years. The risk of major bleeding in patients receiving ACOs is $3 \%$ per year, and approximately $20 \%$ of major bleeds are fatal. Each 1-point increase in the INR increases the risk of major bleeding by $42 \%$. The ACO-related intracranial hemorrhage occurs in a proportion of 2 to 9 per 100,000 population / year, an incidence that is 7-10 times higher than in patients not receiving ACOs. Furthermore, this proportion increases with age [16,21-23].

Thanks to the implementation of CODE STROKE, more patients receive fibrinolytic therapy with rt-PA ev and in some patients 
endovascular rescue therapy is performed. There are few data on the outcome of patients treated with rt-PA or endovascular procedures and what is their risk of intracranial hemorrhage when initiated NACOs treatment in $<14$ days. According to the register SONIIA, which collects and analyzes patients undergoing endovascular procedures in Catalonia the risk of intracranial bleeding is approximately $6 \%$. If renal function is impaired, you should assume that the half-life of dabigatran is elongated. Given this fact, the exclusion window after the last dose for rt-PA should be at least 5 half-lives [24].

Neuroimaging studies prior to initiation of treatment with ACOs can provide clinical information on the risk of ICH associated with indefinite anticoagulant treatment in a given patient and help make the decision as to either indicate or contraindicate treatment. The microbleeds and radiological leukoaraiosis are expressions of underlying vascular disease, vasculopathy amyloid and/or hypertensive, and both have been linked to an increased risk of ICH. The ACOs increase the risk of cerebral hemorrhage in patients with underlying vascular disease [25-27].

Another aspect is concomitant antiplatelet therapy. $40 \%$ of patients in the RE-LY study were taking aspirin at the time of inclusion. In the 3 arms of the study, concomitant treatment with aspirin was associated with an increased risk of bleeding. Concomitant antiplatelet therapy is recommended when maintaining indication for short-term antiplatelet therapy (eg. coronary stents) [18].

In conclusion, in our population older and with higher mean CHAD2DS2-VASc score than in the RELY study, treatment with dabigatran was safe. There were also fewer complications compared with RE-LY. There was no worsening of renal function in patients older than 80 years. These data also support the efficacy and safety of dabigatran in elderly patients. Our sample of patients represents the typical neurological patient population with multiple vascular risk factors and advanced age, which usually have a high CHAD2DS2-VASc and major bleeding risk (HAS-BLED in high range). We believe that this population is even more representative than the RE-LY and with good results in our population to date.

\section{References}

1. Gil Nunez A (2010) Advances in the prevention of cerebral ischaemia due to atrial fibrillation. Neurologia 25: 401-408.

2. Medi C, Hankey GJ, Freedman SB (2010) Stroke risk and antithrombotic strategies in atrial fibrillation. Stroke 41: 2705-2713.

3. Díaz Guzman J (2012) Cardioembolic stroke: epidemiology. Neurologia 27: 4-9.

4. Font MA, Krupinski J, Arboix A (2011) Antithrombotic medication for cardioembolic stroke prevention. Stroke Res Treat 2011:607852.

5. Arboix A, Alio J (2012) Acute cardioembolic cerebral infarction: answers to clinical questions. Curr Cardiol Rev 8: 54-67.

6. Cea-Calvo L, Redón J, Lozano JV, Fernández-Pérez C, Martí-Canales JC, et al. (2007) Prevalence of atrial fibrillation in the Spanish population aged 60 years or more. The PREV-ICTUS study. Rev Esp Cardiol 60: 616-624.

7. Healey JS, Connolly SJ, Gold MR, Israel CW, Van Gelder IC, et al. (2012) Subclinical atrial fibrillation and the risk of stroke. N Engl J Med 366: 120-129.

8. Wyse DG, Waldo AL, DiMarco JP, Domanski MJ, Rosenberg Y, et al. (2002) Atrial Fibrillation Follow-up Investigation of Rhythm Management (AFFIRM) Investigators. A comparison of rate control and rhythm control in patients with atrial fibrillation. N Engl J Med 347: 1825-1833.

9. Martí-Fàbregas J, Delgado-Mederos R, Mateo J (2012) Limitations of anticoagulant therapy. Neurologia 27: 27-32.

10. Gil Núñez A (2012) Prevention of cardioembolic stroke. Neurologia 27: 15-26.

11. Connolly SJ, Ezekowitz MD, Yusuf S, Eikelboom J, Oldgren J (2009) Dabigatran versus warfarin in patients with atrial fibrillation. N Engl J Med 361: 1139-1151.
12. Diener HC, Connolly SJ, Ezekowitz MD, Wallentin L, Reilly PA, et al. (2010) Dabigatran compared with warfarin in patients with atrial fibrillation and previous transient ischaemic attack or stroke: a subgroup analysis of the RELY trial. Lancet Neuro 19: 1157-1163.

13. Schulman S, Majeed A (2012) The oral thrombin inhibitor dabigatran: strengths and weaknesses. Semin Thromb Hemost 38: 7-15.

14. Banerjee A, Lane DA, Torp-Pedersen C, Lip GY (2012) Net clinical benefit of new oral anticoagulants (dabigatran, rivaroxaban, apixaban) versus no treatment in a 'real world' atrial fibrillation population: a modelling analysis based on a nationwide cohort study. Thromb Haemost107: 584-589.

15. Gorter JW (1999) Major bleeding during anticoagulation after cerebra ischemia: patterns and risk factors. Stroke Prevention in Reversible Ischemia Trial (SPIRIT). European Atrial Fibrillation Trial (EAFT) study groups. Neurology 53: $1319-1327$.

16. Greenberg SM, Eng JA, Ning M, Smith EE, Rosand J (2004) Hemorrhage burden predicts recurrent intracerebral hemorrhage after lobar hemorrhage. Stroke 35: 1415-1420.

17. Masjuan J (2012) Aplicaciones de dabigatrán en neurologia. Suplementos Neurología 27: 1-39.

18. Alberts MJ, Bernstein RA, Naccarelli GV, Garcia DA (2012) Using dabigatran in patients with stroke: a practical guide for clinicians. Stroke 43: 271-279.

19. Huisman MV, Lip GY, Diener HC, Brueckmann M, van Ryn J, et al. (2012) Dabigatran etexilate for stroke prevention in patients with atrial fibrillation: resolving uncertainties in routine practice. Thromb Haemost 107: 838-847.

20. Banerjee A, Marín F, Lip GY (2011) A new landscape for stroke prevention in atrial fibrillation: focus on new anticoagulants, antiarrhythmic drugs and devices. Stroke 42: 3316-3322.

21. Rosand J, Hylek EM, O'Donnell HC, Greenberg SM (2000) Warfarin-associated hemorrhage and cerebral amyloid angiopathy: a genetic and pathologic study. Neurology 55: 947-951.

22. Schmidt R, Enzinger C, Ropele S, Schmidt H, Fazekas F (2003) Progression of cerebral white matter lesions: 6-year results of the Austrian Stroke Prevention Study. Lancet 361: 2046-2048.

23. Wang TJ, Massaro JM, Levy D, Vasan RS, Wolf PA, et al. (2003) A risk score for predicting stroke or death in individuals with new-onset atrial fibrillation in the community: the Framingham Heart Study. JAMA 290: 1049-1056.

24. Abilleira S, Dávalos A, Chamorro A, Alvarez-Sabín J, Ribera A, et al. (2011) Catalan Outcomes of intravenous thrombolysis after dissemination of the stroke code and designation of new referral hospitals in Catalonia: the Catalan Stroke Code and Thrombolysis (Cat-SCT) Monitored Study. Stroke Code and Thrombolysis Study Group. Stroke 42: 2001-2006.

25. Fazekas F, Kleinert R, Roob G, Kleinert G, Kapeller P, et al. (1999) Histopathologic analysis of foci of signal loss on gradient-echo $\mathrm{T} 2^{*}$-weighted MR images in patients with spontaneous intracerebral hemorrhage: evidence of microangiopathy-related microbleeds. AJNR Am J Neuroradiol 20: 637-642.

26. Fernandez-Cadenas I, Mendioroz M, Domingues-Montanari S, Del RioEspinola A, Delgado P, et al. (2011) Leukoaraiosis is associated with genes regulating blood-brain barrier homeostasis in ischaemic stroke patients. Eur $\mathrm{J}$ Neurol 18: 826-835.

27. Rybinnik I, Mullen MT, Messe S, Kasner SE, Cucchiara B (2013) Treatment of Acute Stroke in Patients on Dabigatran: A Survey of US Stroke Specialists. J Stroke Cerebrovasc Dis. 